\title{
Dimuon production by laser-wakefield accelerated electrons
}

\author{
A. I. Titov, ${ }^{1,2,3}$ B. Kämpfer, ${ }^{1,4}$ and H. Takabe ${ }^{3}$ \\ ${ }^{1}$ Forschungzentrum Dresden-Rossendorf, 01314 Dresden, Germany \\ ${ }^{2}$ Bogoliubov Laboratory of Theoretical Physics, JINR, Dubna 141980, Russia \\ ${ }^{3}$ Institute of Laser Engineering, Yamada-oka, Suita, Osaka 565-0871, Japan \\ ${ }^{4}$ Institut für Theoretische Physik, TU Dresden, 01062 Dresden, Germany
}

(Received 17 July 2009; published 11 November 2009)

\begin{abstract}
We analyze $\mu^{+} \mu^{-}$pair production generated by high-energy electrons emerging from a laserwakefield accelerator. The $\mu^{+} \mu^{-}$pairs are created in a solid thick high- $Z$ target, following the electron accelerating plasma region. Numerical estimates are presented for 1 to $10 \mathrm{GeV}$ electron beams which are expected to be reliable in the nearest future. Reactions induced by the secondary bremsstrahlung photons dominate the dimuon production. According to our estimates, a $20 \mathrm{pC}$ electron bunch with energy of 1 (10) GeV may create about 100 (5000) muon pairs. The produced $\mu^{ \pm}$can be used in studying various aspects of muon-related physics in tabletop installations. This may be considered as an important step towards the investigation of more complicated elementary processes induced by laser-driven electrons.
\end{abstract}

DOI: 10.1103/PhysRevSTAB.12.111301

PACS numbers: 12.20.Ds, 13.60.Le, 41.75.Jv

\section{INTRODUCTION}

The possibility to produce strong electric fields of the order of $10-100 \mathrm{GV} / \mathrm{m}$ with present laser facilities is a great advantage for laser-wakefield accelerators [1] which allows, in principle, to construct compact accelerating devices for particle and nuclear physics. The successful production of high-quality electron beams in such laserdriven accelerators with electron energies of the order of $1 \mathrm{GeV}$ has been reported recently [2-4]. Electron beams with energies exceeding $1 \mathrm{GeV}$ are interesting for many applications in particle physics, such as investigating the properties and production mechanisms of vector and exotic scalar mesons in photoproduction/electroproduction [5,6], excitation of baryon resonances with the aim of studying their properties and search for missing resonances [7], strangeness photoproduction [8], etc. Another subject is related to the neutrino physics. For example, for studying neutrino oscillations it would be attractive to have two types of neutrinos with fixed intensity. This may be obtained in muon decays $\mu^{+} \rightarrow e^{+}+\nu_{e}+\bar{\nu}_{\mu}$ and $\mu^{-} \rightarrow$ $e^{-}+\bar{\nu}_{e}+\nu_{\mu}$, where muons and electrons neutrinos (or antineutrinos) are produced in equal parts. Therefore, it is interesting to estimate whether the high-energy laserdriven electrons can produce a sizable amount of muon pairs for future applications.

Recall that the present generation of the high-energy neutrino beams are made by decays of charged pions and kaons in flight in a long decay channel. In this case, the neutrino (antineutrino) beams mostly consist of $\nu_{\mu}\left(\bar{\nu}_{\mu}\right)$ with a rather small admixture of electron neutrinos. The idea to use a storage ring of muons as a source of highenergy and high-intensity neutrino beam has been discussed in Refs. $[9,10]$. It is assumed that the muons are produced in two-body decays $\pi^{+} \rightarrow \mu^{+}+\nu_{\mu}$ and $K^{+} \rightarrow$ $\mu^{+}+\nu_{\mu}$ and then stored in a ring with subsequent decay into an electron and two neutrinos. The pions (kaons) in turn are produced in proton-proton and (or) in protonnucleus collisions with high-intensity proton beams [11]. Together with neutrino oscillation, such high-intensity muon sources may be used in studying other fundamental problems of lepton physics, say the search for lepton flavor violation [12] and the measurement of the muon's anomalous magnetic moment [13]. The idea to use a laser-driven proton beam for investigating different aspects of the neutrino oscillations was discussed for the first time in Ref. [14].

The aim of present paper is to analyze the possibility of muon pair creation in the interaction of high-energy laserdriven electrons within a heavy (high- $Z$ ) target in a tabletop configuration. The electromagnetic sources of the $\mu^{+} \mu^{-}$(dimuon) production are described by the following elementary processes:

$$
\begin{gathered}
\gamma+A \rightarrow A+\mu^{+} \mu^{-}, \\
e+A \rightarrow e^{\prime}+A+\mu^{+} \mu^{-} .
\end{gathered}
$$

In the first case (1) the dimuons are produced in the interaction of real (bremsstrahlung) photons within the electric field of the high- $Z$ target nuclei. This is an analog of the well-known Bethe-Heitler process of the electronpositron production. In the second case (2), the dimuons are produced in the interaction of high-energy electrons with nuclei (so-called trident process). These two reactions are depicted in Fig. 1. In some sense these reactions are similar to the dielectron production by laser-driven relativistic electrons at few dozen $\mathrm{MeV}$ analyzed by Nakashima and Takabe [15] and other authors (see, for example, Ref. [16]). Therefore, our investigation may be considered as a continuation of these studies, but with the focus on 


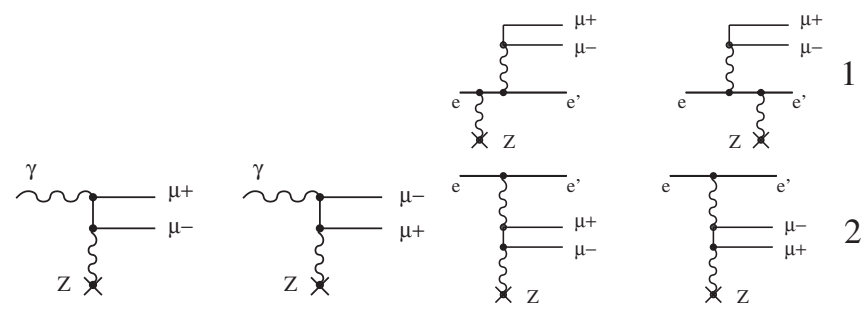

(a)

(b)

FIG. 1. Diagrammatic representation of dimuon production processes in electromagnetic interactions. (a) Bethe-Heitler process $\gamma+A \rightarrow A+\mu^{+} \mu^{-}$. (b) Electron induced reaction $e+$ $A \rightarrow e+A+\mu^{+} \mu^{-}$.

dimuon production. Muon pair creation by two x-ray laser photons was discussed in Ref. [17]. Dimuon production in electron-positron-photon plasmas was estimated in [18].

Our paper is organized as follows. In Sec. II, we recall the relevant elementary electromagnetic processes. Estimates of the dimuon yield are presented in Sec. III, where the energy loss and attenuation of the primary electron beam and the secondary photon bremsstrahlung spectrum are accounted for. A brief discussion of the hadronic sources of muons and the summary are given in Sec. IV.

\section{ELEMENTARY CROSS SECTIONS}

By making use of a standard technique [19], one can express the differential cross section of the dimuon production in the reaction Eq. (1) in the following way:

$$
\begin{aligned}
d \sigma^{\gamma A}= & \frac{Z^{2} \alpha^{3}}{4 \pi} \frac{|\mathbf{q}|}{|\mathbf{k}|}\left|T^{\gamma A}\right|^{2} \sqrt{M_{\mu^{+} \mu^{-}}^{2}-4 \mu^{2}} d M_{\mu^{+} \mu^{-}} d \\
& \times \cos \theta_{q} d \Omega_{\mu^{+}},
\end{aligned}
$$

where $\mathbf{k}$ and $\mathbf{q}$ are the spatial parts of the incoming photon momentum and the total momentum of the outgoing muon pair $q=p_{\mu^{+}}+p_{\mu^{-}}$with the invariant mass of the muon pair $M_{\mu^{+} \mu^{-}}^{2}=q^{2} ; \mu$ denotes the muon mass, $\theta_{q}$ is the polar angle of the direction of flight of dimuon, $\Omega_{\mu^{+}}$is the solid angle of the direction of the velocity of the $\mu^{+}$meson in the rest frame of the dimuon; $Z$ and $\alpha$ stand for the nuclear charge and the fine structure constant $(\alpha=$ $1 / 137)$, respectively; $T^{\gamma A}$ is the invariant amplitude and averaging over photon polarization and summation over $\mu^{ \pm}$spin states is provided in $\left|T^{\gamma A}\right|^{2}$. The quantization axis $z$ is chosen along the velocity of the incoming photon, and the production plane is defined by the vectors $\mathbf{k}$ and $\mathbf{q}$. The differential cross section depends on the initial energy and four kinematical variables, which define the final state.

In the case of the $e+A \rightarrow e^{\prime}+A+\mu^{+} \mu^{-}$reaction, Eq. (2), we have two relevant subprocesses. One corresponds to the electron scattering off the nucleus. In the second case, one of the outgoing muons interacts with the atomic nucleus. These two cases are marked in Fig. 1(b) as
" 1 " and " 2 ", respectively. The total amplitude is the coherent sum of all four amplitudes. The differential cross section for this reaction depends on seven kinematical variables, which define the final state of the outgoing leptons and recoil nucleus, and it has the form (cf. [19])

$$
\begin{aligned}
d \sigma^{e A}= & \frac{Z^{2} \alpha^{4}}{16 \pi^{3}} \frac{\left|\mathbf{p}_{e^{\prime}}\right|}{\left|\mathbf{p}_{e}\right|}\left|T^{e A}\right|^{2} \sqrt{M_{\mu^{+} \mu^{-}}^{2}-4 \mu^{2}}|\mathbf{q}| d E_{q} d M_{\mu^{+} \mu^{-}} d \\
& \times \cos \theta_{e^{\prime}} d \Omega_{q} d \Omega_{\mu^{+}},
\end{aligned}
$$

where $\mathbf{p}_{e}$ and $\mathbf{p}_{e^{\prime}}$ are three momenta of the incoming and outgoing electrons, respectively, $\theta_{e^{\prime}}$ is the polar angle of the direction of flight of the outgoing electron, $E_{q}=$ $\sqrt{\mathbf{q}^{2}+M_{\mu^{+} \mu^{-}}^{2}}$ is the energy of the muon pair; $T^{e A}$ is the invariant amplitude and averaging over initial electron spin projections and summation over the final fermion spin states is understood in $\left|T^{e A}\right|^{2}$; the quantization axis $(\mathbf{z})$ is along the velocity of the incoming electron, and $\mathbf{y}=\left[\mathbf{p}_{\mathbf{e}} \times\right.$ $\left.\mathbf{p}_{\mathbf{e}^{\prime}}\right] /\left|\mathbf{p}_{\mathbf{e}} \| \mathbf{p}_{\mathbf{e}^{\prime}}\right|$.

The invariant amplitudes for reactions in Eqs. (1) and (2) are calculated in the lowest order of the perturbation theory analog to the electron-positron production in $\gamma A$ and $e A$ interactions [20]. The amplitude for the $\gamma A$ reaction reads

$$
\begin{aligned}
T^{\gamma A}= & -\frac{1}{\kappa_{\gamma}^{2}} \bar{u}_{\mu^{-}}\left[\gamma_{0} \frac{g_{1}+\mu}{g_{1}^{2}-\mu^{2}} \gamma_{\alpha}\right. \\
& \left.-\gamma_{\alpha} \frac{g_{2}-\mu}{g_{2}^{2}-\mu^{2}} \gamma_{0}\right] v_{\mu^{+}} \varepsilon_{\gamma}^{\alpha},
\end{aligned}
$$

where $u$ and $v$ are the Dirac spinors of the outgoing $\mu^{-}$and $\mu^{+}$muons, respectively, $\varepsilon_{\gamma}$ is the polarization vector of incoming photons, $g_{1}=k-p_{\mu^{+}}, g_{2}=k-p_{\mu^{-}}$, and $\kappa_{\gamma}=k-q$. The notation $\not p$ means the four product $\gamma_{\alpha} p^{\alpha}$, where $\gamma_{\alpha}(\alpha=0,1,2,3)$ are Dirac's $\gamma$ matrices.

Correspondingly, for the $e A$ reaction we have

$$
T^{e A}=T_{1}^{e A}+T_{2}^{e A}
$$

with

$$
\begin{aligned}
T_{1}^{e A}= & -\frac{1}{\kappa_{e}^{2} q^{2}}\left(\overline { u } _ { e ^ { \prime } } \left[\gamma_{0} \frac{\not p_{1}+m_{e}}{p_{1}^{2}-m_{e}^{2}} \gamma_{\alpha}\right.\right. \\
& \left.\left.-\gamma_{\alpha} \frac{\not p_{2}+m_{e}}{p_{2}^{2}-m_{e}^{2}} \gamma_{0}\right] u_{e}\right)\left(\bar{u}_{\mu^{-}} \gamma^{\alpha} v_{\mu^{+}}\right), \\
T_{2}^{e A}= & -\frac{1}{\kappa_{e}^{2} q^{\prime 2}}\left(\bar{u}_{e^{\prime}} \gamma^{\alpha} u_{e}\right)\left(\overline { u } _ { \mu ^ { - } } \left[\gamma_{0} \frac{p_{3}+\mu}{p_{3}^{2}-\mu^{2}} \gamma_{\alpha}\right.\right. \\
& \left.\left.-\gamma_{\alpha} \frac{\not p_{4}-\mu}{p_{4}^{2}-\mu^{2}} \gamma_{0}\right] v_{\mu^{+}}\right),
\end{aligned}
$$

where $q^{\prime}=p_{e}-p_{e^{\prime}}, p_{1}=p_{e}-q, p_{2}=p_{e^{\prime}}+q, p_{3}=$ $q^{\prime}-p_{\mu^{+}}, p_{4}=q^{\prime}-p_{\mu^{-}}$, and $\kappa_{e}=q^{\prime}-q$.

The effects of nuclear size and higher-order Coulomb corrections are small [21]; the effects of the atomic elec- 
tron screening [22,23] are neglected here as the production processes happen in the high-field region near the target nuclei. The corresponding cross sections, Eqs. (3) and (4), are evaluated numerically without any additional approximation.

The invariant-mass distributions for $\gamma A$ and $e A$ reactions are shown in Fig. 2. In this case, the corresponding cross sections are calculated as a function of the invariant mass $M_{\mu^{+} \mu^{-}}$integrating over the other variables in Eqs. (3) and (4). All calculations are done for a gold target. The initial electron energies are chosen to be 1 and $10 \mathrm{GeV}$. For $1 \mathrm{GeV}$ we follow the laser accelerator conditions of Ref. [2]. The case of $10 \mathrm{GeV}$ may be considered as a prediction for future high-energy tabletop electron accelerators being under consideration now [24,25]. For simplicity, in the case of the $\gamma A$ reaction, the elementary cross sections are calculated at $E_{\gamma}=1$ and $10 \mathrm{GeV}$. Below, for the estimate of the dimuon yield, we fold the elementary cross section with the bremsstrahlung photon distribution for all kinematically allowed photon energies.

Figure 2 demonstrates that the cross section of the electron induced reaction is smaller by 2 orders of magnitude than the corresponding cross section in the photon induced reaction. This is mainly because of an additional vertex with additional factor $\alpha$. Both distributions exhibit a maximum slightly above $\sim 2 \mu$. This means that the relative kinetic energy of the two muons in a pair is small, i.e., they flight close to each other.

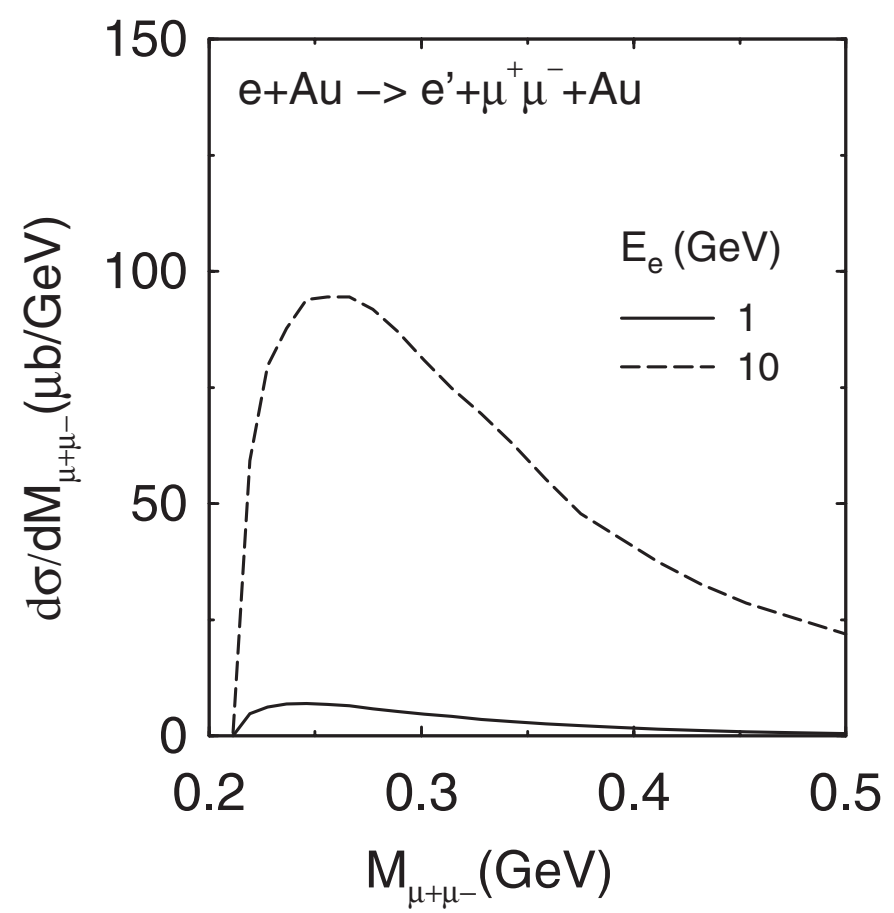

The differential cross sections as a function of the polar angle of the direction of flight of the muon pair $\theta_{q}$ integrated over the other variables are shown in Fig. 3. One can see that the cross sections are peaked at the forward direction, especially for $E_{e, \gamma}=10 \mathrm{GeV}$. The knowledge of the spatial structure of the outgoing muon flux is important for calculating the differential dimuon yield from thicker targets.

The differential cross section of the reaction $e+A \rightarrow$ $e^{\prime}+\mu^{+} \mu^{-}+A$ as a function of the dimuon energy $E_{q}$ is presented in Fig. 4. One can see a wide spread of this distribution with a maximum around $1 \mathrm{GeV}$ for initial electron energy of $10 \mathrm{GeV}$, while for $1 \mathrm{GeV}$ electrons the dimuon energy displays a plateau above $0.6 \mathrm{GeV}$ until the kinematical limit. Since the kinetic energy of the relative motion of $\mu^{+}$and $\mu^{-}$within a pair is small $\left(E_{\mu^{ \pm}} \simeq\right.$ $E_{q} / 2$ ), the energy distribution of the individual muons may be approximated as $d \sigma / d E_{\mu^{+}} \simeq d \sigma / d E_{\mu^{-}} \simeq$ $d \sigma /\left.d E_{q}\right|_{E_{\mu^{ \pm}}=E_{q} / 2}$.

The total cross sections of the dimuon production as a function of the initial energy are shown in Fig. 5. One can see a steep increase of the cross sections with energy. We note that in the ultrarelativistic case with $E_{\gamma} \gg \mu$ the total cross section of the reaction $\gamma A \rightarrow \mu^{+} \mu^{-} A$ may be described by [20]

$$
\sigma \simeq \frac{28}{9} Z_{A}^{2} \alpha r_{0}^{\mu 2}\left(\ln \frac{2 E_{\gamma}}{\mu}-\frac{109}{42}\right)
$$

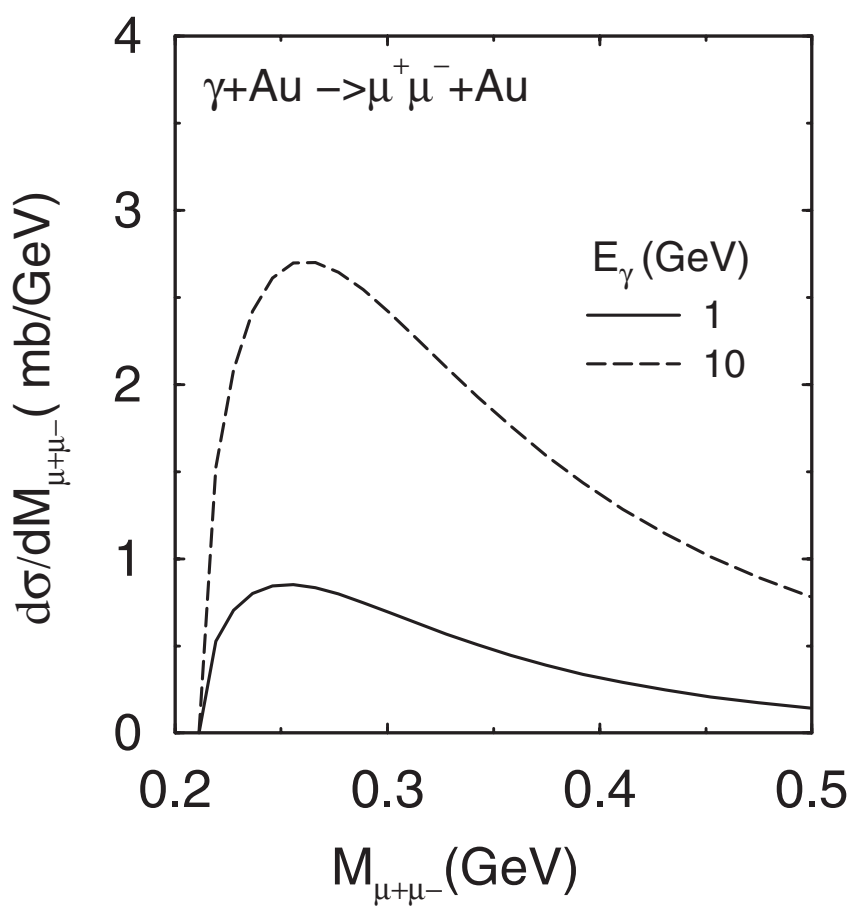

FIG. 2. Differential cross sections of dimuon production in $e A$ (left panel) and $\gamma A$ (right panel) interactions as a function of the invariant dimuon mass $M_{\mu^{+} \mu^{-}}$. Results in left (right) panel are expressed in $\mu \mathrm{b} / \mathrm{GeV}(\mathrm{mb} / \mathrm{GeV}$ ). The solid and dashed curves correspond to initial energies of 1 and $10 \mathrm{GeV}$, respectively. 

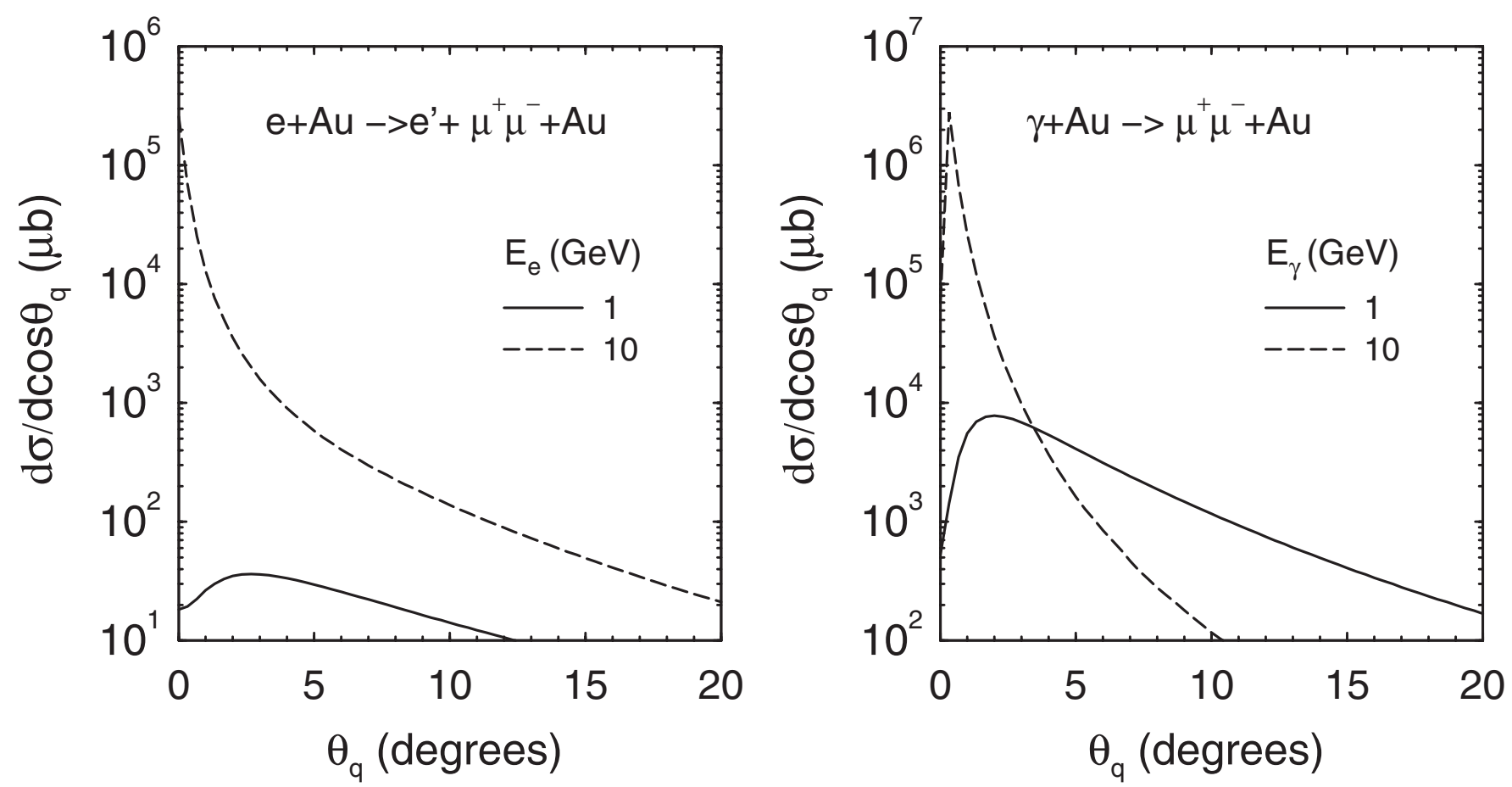

FIG. 3. Differential cross sections of dimuon production in $e A$ (left panel) and $\gamma A$ (right panel) reactions as a function of polar angle $\theta_{q}$. The solid and dashed curves correspond to initial energies 1 and $10 \mathrm{GeV}$, respectively.

where $r_{0}^{\mu}=\alpha / \mu$ is the classical muon radius. The corresponding cross section is shown in Fig. 5 by the dotted curve showing that the ultrarelativistic behavior sets in at $E_{\gamma} \gtrsim 3 \mathrm{GeV}$.

The total cross sections for $\gamma A$ and $e A$ reactions reach $300 \mu \mathrm{b}$ and $3 \mu \mathrm{b}$, respectively, at $E_{\gamma(e)} \simeq 2 \mathrm{GeV}$.

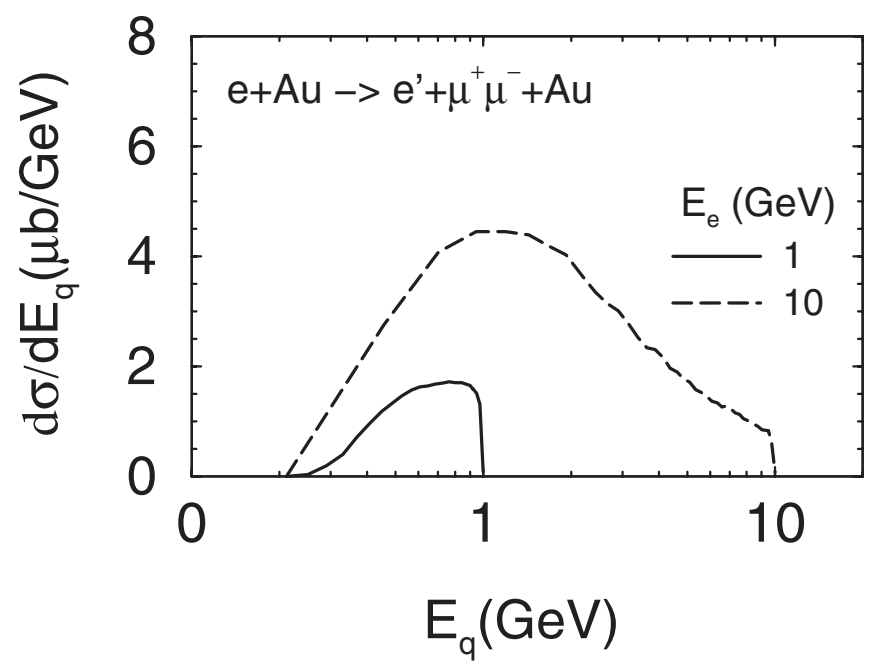

FIG. 4. The differential cross section for electron induced muon pair production reaction as a function of dimuon energy $E_{q}$. The solid and dashed curves correspond to initial energies 1 and $10 \mathrm{GeV}$, respectively.

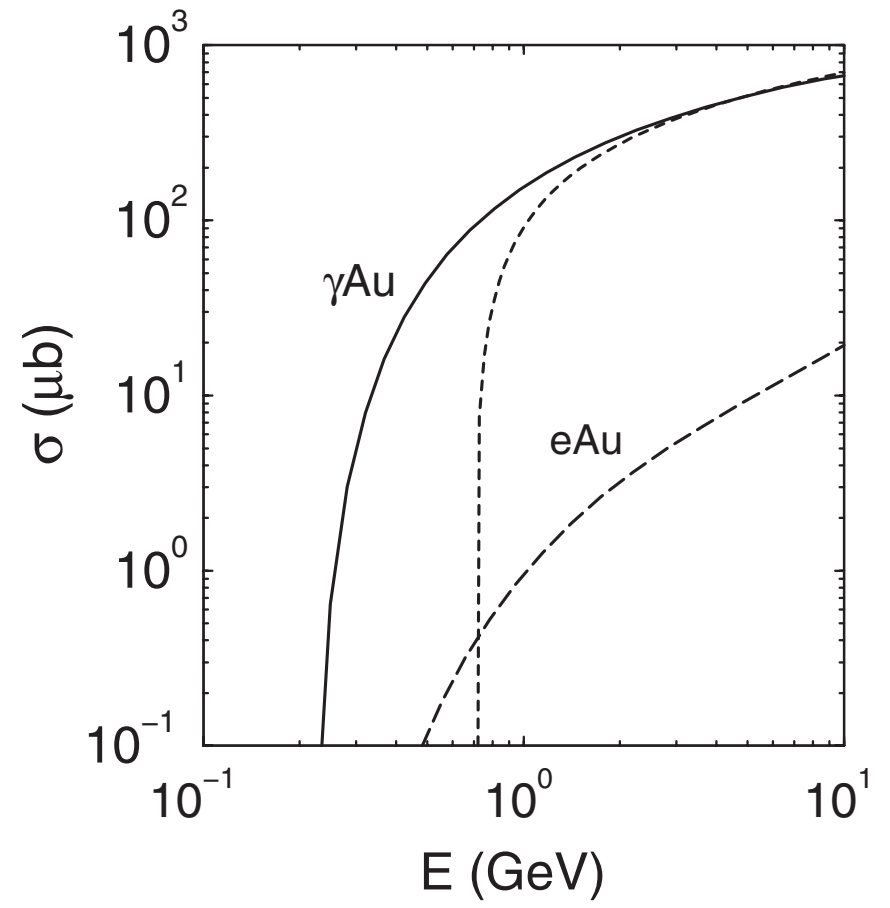

FIG. 5. The total cross section of the dimuon production in $\gamma A$ (solid curve) and $e A$ (dashed curve) reactions as a function of initial energy. The dotted curve corresponds to the high-energy approximation, Eq. (8). 
Similarly to the differential cross sections, the difference between the two reactions is about 2 orders of magnitude, mainly due to an additional power of $\alpha$ in the $e A$ cross section.

\section{DIMUON YIELDS}

Using the above elementary cross sections, one can estimate the dimuon yield for given electron beam and target properties. For the former ones we use the conditions of electron beams as reported for the laser-wakefield accelerator in Ref. [2]. The electron energy is about 0.5$1 \mathrm{GeV}$ and the electron flux is about $20 \mathrm{pC}$ which corresponds to $N_{0}^{e} \simeq 1.248 \times 10^{8}$ electrons in a bunch. In our estimates we assume the same flux for electron energies up to $10 \mathrm{GeV}$. We consider a gold target with thickness of $L=$ $0.1-1 \mathrm{~cm}$.

Strictly speaking, the particle production in interactions of the high-energy electrons with heavy target nuclei must be evaluated by transport-kinetic models (see, for example, Refs. [15,16]). However, for a first qualitative estimate of the dimuon yield, one can use an analytical approach, similar to that developed in Ref. [26].

Consider first the dimuon yield which stems from elementary $e A \rightarrow e^{\prime}+\mu^{+} \mu^{-}+A$ reactions. It may be expressed as

$$
d N^{\mu^{+} \mu^{-}}=\frac{N_{A} \rho_{A}}{A} \int_{0}^{L} d l N^{e}(l) d \sigma^{e A \rightarrow e^{\prime} \mu^{+} \mu^{-} A}\left[E_{e}(l)\right],
$$

where $A$ is the atomic weight, $N_{A}$ is Avogadro's number, $\rho_{A}$ denotes the target density, and $d \sigma^{e A \rightarrow e^{\prime} \mu^{+} \mu^{-} A}$ is the elementary cross section of the dimuon production discussed above. For the sake of simplicity, we neglect the energy spread in the bunch taking the electron energy at the central positions of the energy distribution. This seems to be reasonable, because the energy spreading reported in [2] is less than $100 \mathrm{MeV}$ at $E_{e} \simeq 1 \mathrm{GeV}$.

Propagating through the target material, the electron beam loses its intensity and energy. The energy loss is described by [20]

$$
\frac{d E_{e}}{d l}=-E_{e} \frac{N_{A} \rho_{A} \alpha Z_{A}^{2} r_{0}^{e 2}}{A}\left(4 \ln \frac{183}{Z_{A}^{1 / 3}}+\frac{2}{9}\right)
$$

with $r_{0}^{e}=\alpha / m_{e} \simeq 2.818 \times 10^{-13} \mathrm{~cm}$. For the gold target with $A=197$ and $Z_{A}=79$ this leads to the formula

$$
E_{e}(l) \simeq E_{e}^{0} \exp \left(-l / l_{0}\right)
$$

with $l_{0} \simeq 0.306 \mathrm{~cm}$. The electron beam absorption in the target may be accounted for by a linear dependence

$$
N^{e}(l) \simeq N_{0}^{e}\left[1-l / L_{\max }\left(E_{e}^{0}\right)\right],
$$

where $N_{0}^{e}$ is the initial beam intensity and $L_{\max }$ stands for the maximum distance traveled by the high-energy electrons. For the gold target, one can use $L_{\max } \simeq 1.44 \mathrm{~cm}$ at $E_{e}=0.5 \mathrm{GeV}$ which may be extrapolated as $L_{\max }(E) \simeq$
$0.23 E^{0.3} \mathrm{~cm}$ with $E$ in $\mathrm{GeV}$ [27]. Both the decrease of the beam intensity and the energy loss lead to a decrease of the effectiveness of the dimuon production in the target material. However, the effect of the energy loss is significantly greater than the effect of intensity depletion since the scale parameter $l_{0}$ in Eq. (11) is much smaller than $L_{\max }$.

The yield of dimuons, produced by secondary real photons created at the distance $l$ behind the target front side, reads

$$
\begin{aligned}
d N^{\mu^{+} \mu^{-}}= & \frac{N_{A} \rho_{A}}{A} \int_{0}^{L} d l \int d E_{\gamma} \frac{d N_{\gamma}(l)}{d E_{\gamma}} \\
& \times(L-l) d \sigma^{\gamma A \rightarrow \mu^{+} \mu^{-} A}\left(E_{\gamma}\right),
\end{aligned}
$$

where $d \sigma^{\gamma A \rightarrow \mu^{+} \mu^{-} A}$ is the above cross section of the dimuon production in the elementary $\gamma A$ interaction, and $d N_{\gamma}(l) / d E_{\gamma}$ denotes the distribution of bremsstrahlung photons with energy $E_{\gamma}$ at distance $l$ :

$$
\frac{d N_{\gamma}(l)}{d E_{\gamma}}=\frac{N_{A} \rho_{A}}{A} N^{e}(l) \frac{d \sigma_{\gamma}\left[E_{e}(l), E_{\gamma}\right]}{d E_{\gamma}},
$$

where $N^{e}(l)$ is defined by Eq. (12) and the current electron energy $E_{e}(l)$ from Eq. (11) is to be used. For the angularintegrated bremsstrahlung cross section $d \sigma_{\gamma}\left(E_{e}, E_{\gamma}\right) / d E_{\gamma}$, we adopt a parametrization motivated by the analytical expression of Ref. [20]:

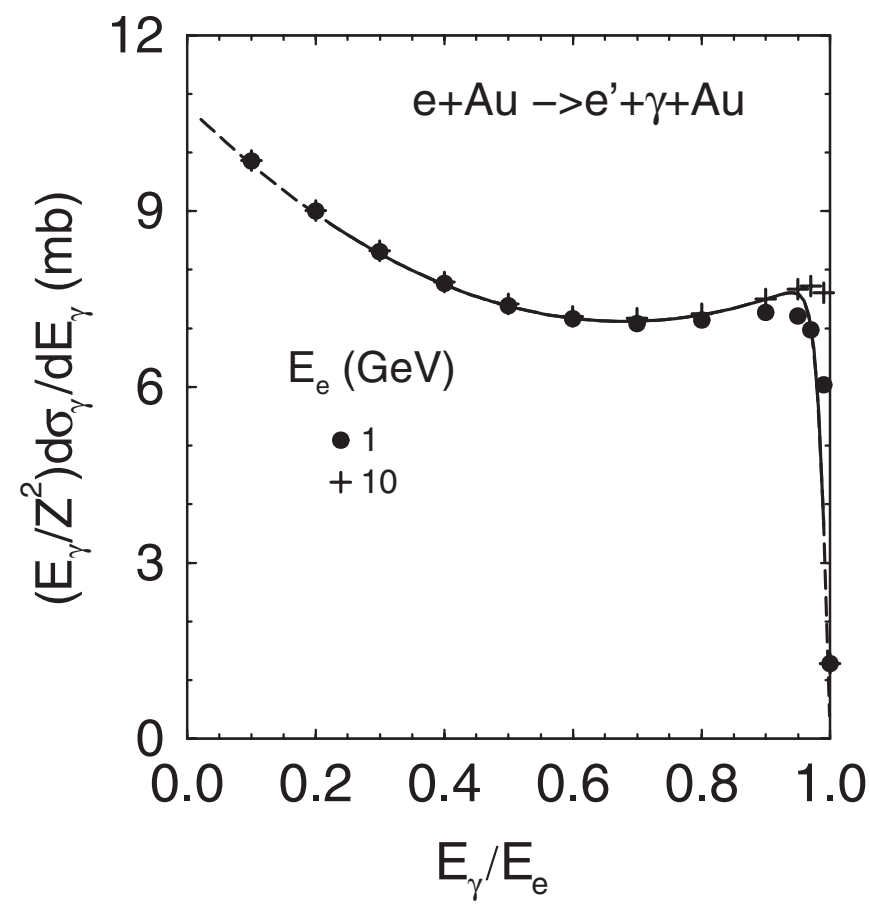

FIG. 6. The scaled bremsstrahlung cross section $\left(E_{\gamma} / Z^{2}\right) d \sigma_{\gamma}\left(E_{e}, E_{\gamma}\right)$ as a function of $E_{\gamma} / E_{e}$ for two electron energies $1 \mathrm{GeV}$ (solid curve) and $10 \mathrm{GeV}$ (dashed curve). The symbols represent the data compilation of Ref. [28]. 

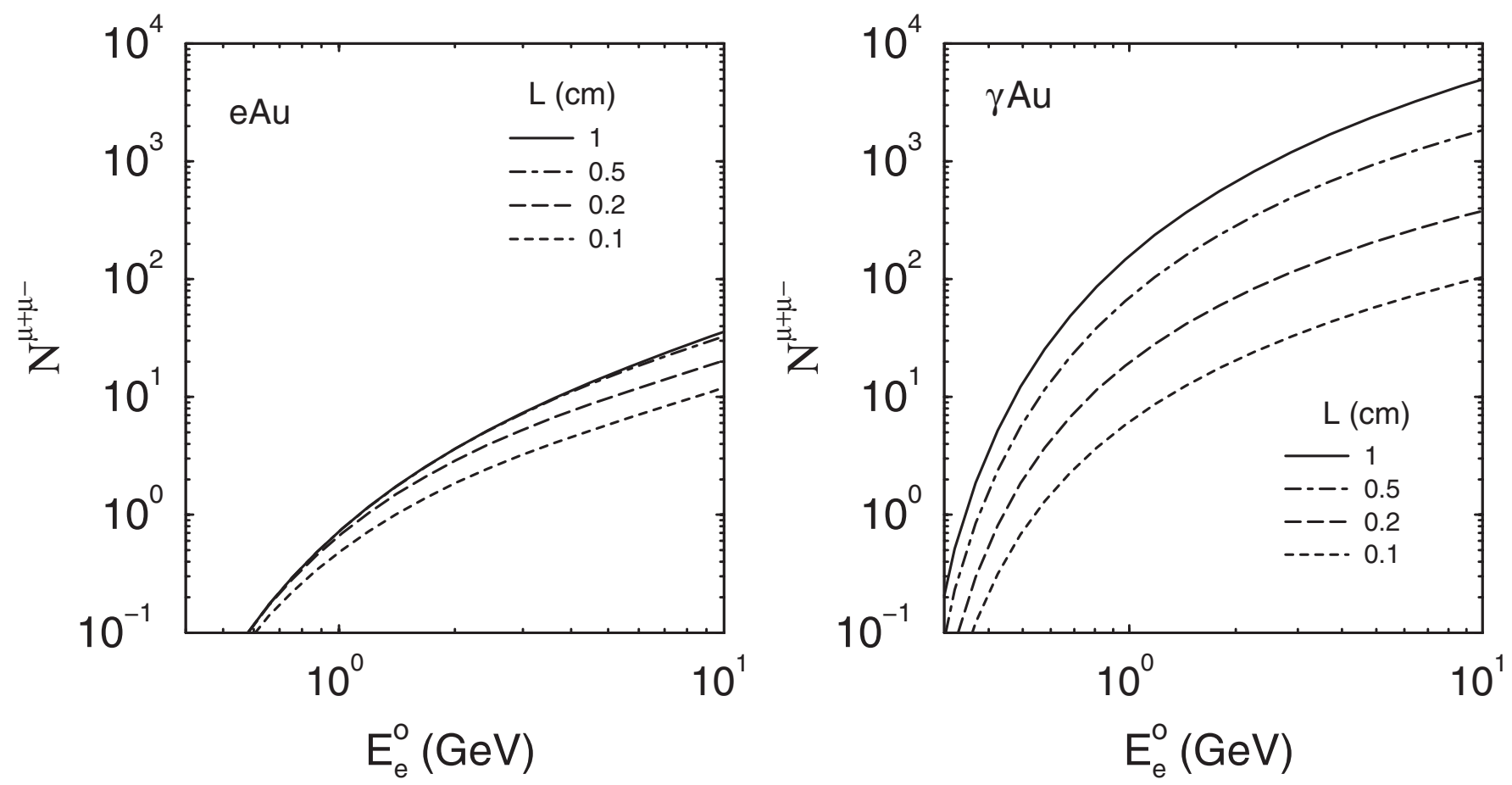

FIG. 7. The yields of dimuons in $e A$ (left panel) and $\gamma A$ (right panel) interactions as a function of primary electron energy $E_{e}^{0}$, calculated by Eqs. (9) and (13), respectively.

$$
\begin{aligned}
\frac{E_{\gamma}}{Z_{A}^{2}} \frac{d \sigma_{\gamma}}{d E_{\gamma}}= & \frac{4 \alpha r_{0}^{e 2} F\left(E_{e}, E_{\gamma}\right)}{E_{e}^{2}}\left[\left(E_{e}^{2}+E_{e^{\prime}}^{2}-\frac{2}{3} E_{e} E_{e^{\prime}}\right)\right. \\
& \left.\times \ln 183 Z_{A}^{-1 / 3}+\frac{E_{e} E_{e^{\prime}}}{9}\right],
\end{aligned}
$$

where $\quad E_{e^{\prime}}=E_{e}-E_{\gamma} \quad$ and $\quad F\left(E_{e}, E_{\gamma}\right)=$ $0.91 \tanh \left(E_{e^{\prime}} / 0.02 E_{e}\right)$. In Fig. 6 we show the bremsstrahlung cross section for two energies together with the compilation of Ref. [28].

Results of calculations of the dimuon yields $N^{\mu^{+}} \mu^{-}$ predicted by Eqs. (9) and (13) as a function of the primary electron energy $E_{e}^{0}$ are exhibited in Fig. 7. The total number of dimuons produced in $e A$ interactions is about 1 and 60 for $E_{e}^{0}=1$ and $10 \mathrm{GeV}$, respectively, for thick target. The yield increases with the thickness of the target. Some saturation sets in at $L \geq L_{\mathrm{sat}} \simeq 0.5 \mathrm{~cm}$. This means that the dimuons are essentially produced in a narrow region of the target mostly because of the large energy loss. It seems to be natural, because the saturation length $L_{\text {sat }}$ is close to the scale parameter $l_{0}$ in Eq. (11).

The number of dimuons in $\gamma A$ interactions is about 100 and 5000 for $E_{e}^{0}=1$ and $10 \mathrm{GeV}$, respectively, for target thickness of $1 \mathrm{~cm}$. The dependence of the total yield on the target thickness is rather strong due to the large main free path of the bremsstrahlung photons.

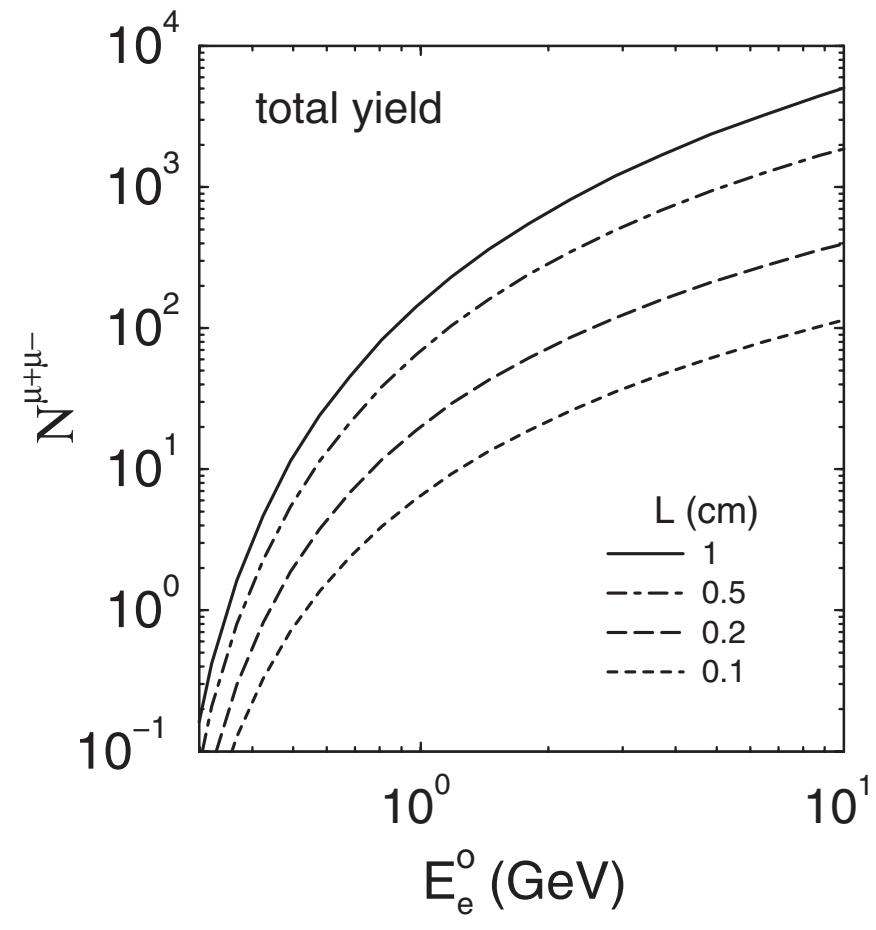

FIG. 8. The total yield of dimuons in interactions of relativistic electrons with the gold target as a function of the primary electron energy $E_{e}^{0}$ and target thickness $L$. 
The dimuon yield in $\gamma A$ interactions considerably exceeds the corresponding yield in $e A$ interactions. This excess strongly depends on the target thickness. Therefore, in Fig. 8 we present the total dimuon yield in interactions of relativistic electrons with a gold target which is a sum of the two above contributions as a function of the primary electron energy $E_{e}^{0}$ and target thickness $L$. For thick targets it practically coincides with the result shown in Fig. 7 (right panel). Finally, note that here we discussed the total dimuon yield. The differential dimuon distributions may be obtained using the differential elementary distributions discussed in Sec. II.

\section{SUMMARY AND DISCUSSION}

In summary we have considered muon pairs production by $\mathrm{GeV}$ electrons, created by a laser-wakefield accelerator, impinging on a thick high- $Z$ target. We estimated the effectiveness of a such a source of muon pairs. For a $1 \mathrm{~cm}$ thick gold target, $1.25 \times 10^{8}$ electrons in a $20 \mathrm{pC}$ bunch with energy of 1 (10) GeV in the initial state produce about $1 \times 10^{2}\left(5 \times 10^{3}\right)$ dimuons with pair energies centered at $1 \mathrm{GeV}$. To get $10^{6}$ dimuons from the muon factory one needs $10^{10}-10^{11}$ primary electrons in a bunch. Such intensities with power of $100 \mathrm{~J}$ seem to be quite realistic in the near future, requiring ultrahigh intensity laser pulses with efficient acceleration mechanisms. Cooling of the heat load in the target material analog to Ref. [29] may be an option for future higher repetition rates. The produced muons, unlike electrons and hadrons, penetrate the target material without suffering noticeable scattering and absorption.

Finally, let us consider the hadronic sources of muons in interactions of the laser-wakefield accelerated electrons with the thick target. The main source of muons here is the photoproduction and/or electroproduction and subsequent decay of $\pi^{ \pm}$mesons in the elementary processes $\gamma+p \rightarrow \pi^{+}+n, \quad \gamma+n \rightarrow \pi^{-}+p, \quad \gamma+N \rightarrow$ $\pi^{+}+\pi^{-}+N$, where $\gamma$ stands for real bremsstrahlung photons (photoproduction) or virtual photons (electroproduction). The production of $K$ mesons by $1 \mathrm{GeV}$ electrons is strongly suppressed because of energy arguments; however, at higher energies they contribute to the muon production similarly to the pions. Charged pions decay via $\pi^{+} \rightarrow \mu^{+}+\nu_{\mu}, \pi^{-} \rightarrow \mu^{-}+\bar{\nu}_{\mu}$ with a probability of 99.99\%. The cross sections of the $\gamma N$ reactions for $E_{\gamma} \sim$ $1 \mathrm{GeV}$ are around $100 \mu \mathrm{b}$ (see, for example, [30]). Pion photoproduction in $\gamma A$ reactions is proportional to $A^{2 / 3}$. This means that the cross section of the muon production at heavy target nuclei by $\gamma A \rightarrow \pi^{ \pm} X \rightarrow \mu^{ \pm}+\nu_{\mu}\left(\bar{\nu}_{\mu}\right) X$ reactions exceeds the dimuon photoproduction, considered in the previous section, by a factor of 20-30. However, taking into account the large pion rescattering and absorption cross sections at heavy target nuclei by strong interaction processes, the resulting pions flux would be weak and not collimated in a thick target. The target must be thin enough to get a well collimated pion/muon beam, but in such a case the intensity of the pion and muon flux will be small. This condition reduces the muon yield considerably. For example, for a dozen $\mu \mathrm{m}$ thick gold target the expected yield of $\mu^{ \pm}$muons is less than one event for the above considered electron bunch. Therefore, the direct production of dimuons in electromagnetic interactions seems to be a more favorable mechanism.

Thus the configuration of a laser-driven electron accelerator and thick high- $Z$ target may serve as an all-optics tabletop device for muon pair production. Their advantage at present is related to the high density of the particles, the excellent normalized emittance, the small size of the driver, possible high power scalability, synergies with nuclear fusion, etc.

The produced $\mu$ mesons may be used in studying various aspects of muon and neutrino physics and be considered as an important step towards investigations of more complicated electron induced elementary processes.

\section{ACKNOWLEDGMENTS}

The authors appreciate S. V. Bulanov, T.E. Cowan, and T. Zh. Esirkepov for fruitful discussions.

[1] T. Tajima and J. M. Dawson, Phys. Rev. Lett. 43, 267 (1979).

[2] W. P. Leemans et al., Nature Phys. 2, 696 (2006).

[3] S. Karsch et al., New J. Phys. 9, 415 (2007).

[4] J. Osterhoff et al., Phys. Rev. Lett. 101, 085002 (2008).

[5] A. I. Titov and T.S.H. Lee, Phys. Rev. C 66, 015204 (2002).

[6] A. Donnachie and Yu. S. Kalashnikova, Phys. Rev. C 78, 064603 (2008).

[7] Y. S. Oh, A. I. Titov, and T. S. H. Lee, Phys. Rev. C 63, 025201 (2001).

[8] B. Saghai, J.-C. David, B. Julia-Diaz, and T.-S. H. Lee, Eur. Phys. J. A 31, 512 (2007).

[9] S. Geer, Phys. Rev. D 57, 6989 (1998); 59, 039903(E) (1999).

[10] S. Geer, J. Phys. G 29, 1485 (2003).

[11] A. Bandyopadhyay et al. (ISS Physics Working Group), arXiv:0710.4947.

[12] W. J. Marciano, T. Mori, and J. M. Roney, Annu. Rev. Nucl. Part. Sci. 58, 315 (2008).

[13] G.W. Bennett et al. (Muon $g-2$ Collaboration), Phys. Rev. D 73, 072003 (2006).

[14] S. V. Bulanov, T. Esirkepov, P. Migliozzi, F. Pegoraro, T. Tajima, and F. Terranova, Nucl. Instrum. Methods Phys. Res., Sect. A 540, 25 (2005).

[15] K. Nakashima and H. Takabe, Phys. Plasmas 9, 1505 (2002).

[16] S. Karsch et al., Laser Part. Beams 17, 565 (1999).

[17] C. Muller, C. Deneke, and C. H. Keitel, Phys. Rev. Lett. 101, 060402 (2008).

[18] I. Kuznetsova, D. Habs, and J. Rafelski, Phys. Rev. D 78, 014027 (2008). 
[19] E. Byckling and K. Kajantie, Particle Kinematics (Wiley, New York, 1973).

[20] A. I. Akhiezer and V.B. Berestetsky, Quantum Electrodynamics (Interscience, New York, 1965).

[21] G. Roche, C. Ducos, and J. Prortol, Phys. Rev. A 5, 2403 (1972).

[22] L. I. Schiff, Phys. Rev. 83, 252 (1951).

[23] E. Borie and H. Arenhoevel, Z. Phys. 255, 459 (1972).

[24] T. Tajima and G. Mourou, Phys. Rev. ST Accel. Beams 5, 031301 (2002).

[25] G. A. Mourou, T. Tajima, and S. V. Bulanov, Rev. Mod.
Phys. 78, 309 (2006).

[26] P. L. Schkolnikov, A. E. Kaplan, A. Pukhov, and J. Meyerter-Vehn, Appl. Phys. Lett. 71, 3471 (1997).

[27] A. P. Babichev et al., Physical Values. Hand-Book, edited by I. S. Grigoriev and E. Z. Meilikhov (Energoiatomizdat, Moscow, 1991) (in Russian).

[28] S. M. Seltzer and M. J. Berger, At. Data Nucl. Data Tables 35, 345 (1986).

[29] J. Klug et al., Nucl. Instrum. Methods Phys. Res., Sect. A 577, 641 (2007).

[30] S. Schadmand, Pramana 66, 877 (2006). 